\title{
PREPARATION AND CHARACTERIZATION OF CEFTRIAXONE SODIUM ENCAPSULATED CHITOSAN NANOPARTICLES
}

\author{
P. MANIMEKALAI ${ }^{*}$, R. DHANALAKSHMI ${ }^{2}$, R. MANAVALAN ${ }^{3}$
}

${ }^{1}$ School of Pharmaceutical Sciences, VELS University, Pallavaram, Chennai 117, ${ }^{2}$ E. G. S. Pillai College of Pharmacy, Nagapattinam 611002, ${ }^{3}$ RVS College of Pharmaceutical Sciences, Sulur, Coimbatore 641402

Email: mekalaivel@gmail.com

Received: 24 Nov 2016, Revised and Accepted: 10 Oct 2017

ABSTRACT

Objective: The objective of this study was to prepare ceftriaxone sodium chitosan nanoparticles (CS-NP) from different drug and polymer ratios and analyze their physicochemical characteristics.

Methods: Ceftriaxone sodium loaded chitosan nanoparticles were prepared using chitosan as a polymer and tri sodium polyphosphate (TPP) as cross linking agent by ionic cross linking and coacervation with the aid of sonication. Various trials have been carried out for the confirmation of nanoformulation. Parameters such as the zeta potential, polydispersity, particle size, entrapment efficiency, in vitro drug release Thermo gravimetric analysis and scanning electron microscope of the nanoparticles were assessed for confirmation of nanoformulation.

Results: The formulated nanoparticles showed mean particle size, polydispersity index and zeta potential to be $183.1 \pm 8.42 \mathrm{~nm}, 0.212 \pm 0.05$, $+38.5 \pm 1.6 \mathrm{mV}$ respectively and the drug loading was found to be $46.42 \pm 10 \%$. In vitro drug release was showed a biphasic release pattern with initial burst release followed by sustained release of formulated nanoparticles. The cumulative percentage of drug release was about $83.08 \%$.

Conclusion: Formulation F2 was found to be the best formulation with a higher cumulative percentage of drug release. Modified ionic gelation method can be utilized for the development of chitosan nanoparticles of ceftriaxone sodium. Polymer and crosslinking agent concentrations and sonication time are rate-limiting factors for the development of the optimized formulation. The chitosan nanoparticles developed would be capable of sustained delivery of ceftriaxone sodium.

Keywords: Ceftriaxone sodium, Chitosan nanoparticles, Zeta potential analysis, Scanning Electron Microscope Analysis, Particle size

(C) 2017 The Authors. Published by Innovare Academic Sciences Pvt Ltd. This is an open access article under the CC BY license (http://creativecommons.org/licenses/by/4.0/) DOI: http://dx.doi.org/10.22159/ijap.2017v9i6.16317

\section{INTRODUCTION}

Ceftriaxone sodium is a semisynthetic antibiotic that can effectively treat several types of bacterial infections. It is a $\beta$ lactamase-resistant cephalosporin with an extremely long serum half-life up to 4-to 10-times longer than the other antibiotics in this class. Because of its poor cellular penetration the effectiveness of antimicrobial treatment is limited especially third-generation cephalosporin. The poor cellular penetration of the antibiotic was associated to its high molecular weight $(661.6 \mathrm{~kg})$ as well as its hydrophilicity $(\log P-0.6)[1]$.

In recent years, it has become more and more evident that the development of new drugs alone is not sufficient to ensure progress in drug therapy. A promising strategy to overcome these problems involves the development of suitable drug carrier systems. Solid nanoparticles were introduced at the beginning of the 1990s, as an alternative to solid nanoparticles, emulsions and liposomes in cosmetic and pharmaceutical preparations [2-5].

Chitosan is a promising nano-carrier to release the encapsulated drug to the targeted tissues. Chitosan nanoparticles can be prepared by the interaction of oppositely charged macromolecules [3]. Compared with other biological polymers, chitosan is one of the most extensively used polymers. This is because chitosan possesses some ideal properties of polymeric carriers for nanoparticles such as biocompatible, biodegradable, nontoxic, and inexpensive [4]. The interaction can be controlled by the charge density of TPP and chitosan, which is dependent on the $\mathrm{pH}$ of the solution. Nano particulate systems show their promise as a potential ideal drug delivery system for poorly soluble, poorly absorbed and labile substances [5]. To overcome the limitation of synthetic drug, targeted drug delivery systems are designed. Nanoparticles have unique functional characters such as small particle size, high stability, lower toxicity, tuneable hydrophilic-hydrophobic balance and the ability to bear surface features for target specific localization, etc. The aim of the present study was to prepare and characterize ceftriaxone sodium loaded chitosan nanoparticles and produced release of ceftriaxone sodium.

\section{MATERIALS AND METHODS}

Ceftriaxone sodium, chitosan (CS) (MW=60-90 k Da; degree of deacetylation 85\%) and sodium tripolyphosphate (TPP) were purchased from Sigma Aldrich, USA. All other chemicals were used analytical grade.

\section{Synthesis of ceftriaxone sodium CS-NP}

The chitosan-TPP nanoparticles were prepared via TPP ionic crosslinking and coacervation method as previously mentioned [8]. The chitosan nanoparticle synthesis procedure was first optimized at different concentrations of chitosan and the resulting mixtures were observed to be either clear, opalescent, or consisting of aggregates.

The polymer was dissolved in $10 \mathrm{ml}$ of $1 \%(\mathrm{v} / \mathrm{v})$ acetic acid. The solution was continuously sonicated at room temperature and the $\mathrm{pH}$ of the solution was adjusted to 5.0 using $1 \mathrm{M} \mathrm{NaOH}$. Ceftriaxone sodium at three different concentrations such as 25, 50 and $100 \mathrm{mg}$ dissolved in water then added to the freshly prepared chi-tosan dispersion and stirred for $1 \mathrm{~h}$. Finally, TPP (1 mg/ml) was added drop wise to the CS-ceftriaxone sodium solution under stirring (750rpm). 10. The resulting mixture was allowed to stir for $4 \mathrm{~h}$ to form ceftriaxone sodium encapsulated chitosan nanoparticles. All the process was carried out under room temperature. The synthesized nanoparticles were isolated by centrifugation. The formulations were then freeze-dried in the presence of $0.1 \%$ sucrose used as a cryoprotectant (Heto Drywinner, Thermo Scientific, USA) for $24 \mathrm{~h}$ at a pressure of $0.05 \mathrm{mmHg}$. The pellets were collected and stored at $4{ }^{\circ} \mathrm{C}$ for further use. 
Table 1: Formulation design for the synthesis of ceftriaxone sodium loaded chitosan nanoparticles

\begin{tabular}{llll}
\hline Formulation code & Amount of drug (mg/ml) & Amount of chitosan (mg) & Amount of TPP (mg/ml) \\
\hline F1 & 10 & 10 & 0.25 \\
F2 & 10 & 20 & 0.25 \\
F3 & 10 & 30 & 0.25 \\
F4 & 10 & 40 & 0.25 \\
F5 & 10 & 50 & 0.25 \\
\hline
\end{tabular}

\section{Percentage practical yield}

The practical yield of chitosan nanoparticles was calculated as follows:

Percentage Yield $=$ weight of Lyophilized nanoparticle X 100 $\div$ Sum of initial dry weight of starting material

\section{Entrapment efficiency (EE) of nanoparticles}

The nanoparticles suspension was centrifuged at $15000 \mathrm{rpm}$ for 30 min. The supernatant solution was separated. $1 \mathrm{ml}$ of supernatant was distributed in $10 \mathrm{ml}$ distilled water and the absorbance was measured using a UV spectrophotometer at $240 \mathrm{~nm}$ using water as blank. The amount of drug unentrapped in the supernatant was calculated. The amount of drug entrapped was determined by subtracting amounts of free, uninterrupted drug from the total amount of ceftriaxone sodium taken [10-12].

The drug entrapment efficiency (EE) of nanoparticles was calculated as follows:

Entrapment Efficiency (\%)

$=$ Total amount of drug- unentrapped drug X 100

$\div$ Total amount of drug

All measurements were performed in triplicate.

\section{In vitro drug release study}

In vitro drug release study was carried out with phosphate buffered solution (PBS) medium according to Dudhani and Kosaraju [18]. The release of ceftriaxone sodium chitosan nano formulation was determined using a modified dialysis membrane diffusion technique [13-17]. An accurately weighed amount of ceftriaxone sodium chitosan nano formulation equivalent to $50 \mathrm{mg}$ of ceftriaxone sodium was transferred to a plastic cylinder having the length of $6 \mathrm{~cm}$ and diameter of $2.5 \mathrm{~cm}$ fitted at its lower end with dialysis membrane (Himedia dialysis membrane with the molecular cut off of $10 \mathrm{kDa}$ ) presoaked with distilled water for $24 \mathrm{~h}$. The cylinder was then suspended in the dissolution flask of a USP dissolution apparatus using $900 \mathrm{ml}$ of phosphate buffer solution ( $\mathrm{pH} 7.2)[19,20]$. The cylinder was allowed to rotate at a constant speed $(25 \mathrm{rpm})$ at $37{ }^{\circ} \mathrm{C} \pm 0.5$. Aliquots were withdrawn and replaced with dissolution media and then the drug content was determined by measuring the absorbance values at 240 $\mathrm{nm}$ using UV spectrophotometric method at predetermined time intervals for a total period of $24 \mathrm{~h}$. All the operations were carried out in triplicate. And the release of ceftriaxone sodium was calculated from the following equations:

$$
\begin{aligned}
\text { Release rate }(\%)= & \text { Released ceftriaxone sodium } \times 100 \\
& \div \text { Total ceftriaxone sodium }
\end{aligned}
$$

\section{UV-visible spectroscopy}

The UV-visible spectra were obtained from UV-visible spectrophotometer, Shimadzu UV-1800 model, Japan. All spectra were collected by recording between 200 and $800 \mathrm{~nm}$ and were corrected against chitosan as a background.

\section{Zeta sizer and zeta potential analysis}

The average particle size and zeta potential of the nanoparticles was determined using zeta sizer Nano series (Malvern Instrument, MAL 1037088, USA). The measurement was carried with ultra-pure water using disposable zeta cells with the general purpose protocol at 25 . The instrument was calibrated routinely with5 $1 \mathrm{mV}$ lat ex standard. The mean zeta potential was determined using phase analysis light scattering technique [22].

\section{Scanning electron microscope analysis [23]}

The morphological features were examined by field emissionscanning electron Microscopy FE-SEM), JEOL, JSM-6700F, Japan. The samples were sprinkled onto a double-sided tape and sputter coated with a $5 \mathrm{~nm}$-thick gold layer [23].

\section{FT-IR analysis}

The FT-IR spectra of the samples were analyzed using a PerkinElmer, FT-IR spectrophotometer, USA within the range of 4000-400 $\mathrm{cm}^{-1}$. About $5 \mathrm{mg}$ of sample was mixed with $100 \mathrm{mg}$ of $\mathrm{KBr}$ and compressed into pellet using a hydraulic press [24]. All spectra were corrected against the reference spectrum of KBr pellet.

\section{Thermo gravimetric analysis}

A Perkin-Elmer Model of TGA-7 thermo gravimetric system with a microprocessor driven temperature control unit and a TA data station was used. The mass of the samples was generally in the range of 2-3 mg. The sample pan was placed in the balance system equipment and the temperature was raised from 25 to $800{ }^{\circ} \mathrm{C}$ at a heating rate of $10{ }^{\circ} \mathrm{C}$ per min with the nitrogen flow rate of $50 \mathrm{~cm}^{3} / \mathrm{min}$. The mass of the sample pan was continuously recorded as a function of temperature [25].

\section{RESULTS AND DISCUSSION}

Synthesis of ceftriaxone sodium loaded chitosan nanoparticles

Chitosan nanoparticles were synthesized by ionic cross linking and coacervation method with a chitosan to TPP ratio of 4:1 as reported in the literature [17]. The successful loading of ceftriaxone sodium CS-NP was optimized by varying the concentrations of ceftriaxone $(10,20,40$, 80 and $100 \mathrm{mg}$ ) and was further confirmed by UV-visible spectrophotometry. Ceftiraxone in acetic acid solution gives major absorption bands at $259 \mathrm{~nm}$ indicating the presence of hydroxyl group and the lactone ring in its structure [21]. However, the UV-visible spectrum of the synthesized ceftriaxone sodium CS-NP exhibited absorption peak at $249 \mathrm{~nm}$ corresponds to the wavelength absorbed in Ceftriaxone sodium. This result indicates the successful loading of ceftriaxone within chitosan nanoparticles. Following the synthesis of ceftriaxone sodium CS-NP, their encapsulation and loading efficiency was calculated based on the UV-visible absorption spectral studies. As shown in table 2 the maximum encapsulation efficiency was determined by varying the concentration of ceftriaxone sodium in CS/TPP ratio (2:1). The polymer concentration of $20 \mathrm{mg}$ showed high encapsulation $86.51 \pm 50$.

Table 2: Entrapment efficiency of ceftriaxone sodium into CS-NP determined by UV-visible spectrophotometry

\begin{tabular}{ll}
\hline Formulation code (drug+polymer ratio) & Entrapment efficiency \\
\hline F1(1:1) & $65.59 \pm 30$ \\
F2 (1:2) & $86.51 \pm 50$ \\
F3 (1:3) & $66.81 \pm 40$ \\
F4(1:4) & $49.70 \pm 90$ \\
F5 (1:5) & $46.42 \pm 10$ \\
\hline
\end{tabular}

Data's are expressed as mean \pm SEM $(n=3)$ 


\section{Manimekalai et al.}

\section{Particle size and zeta potential}

Particle size and surface charge are the major parameters to be focused to get effective drug delivery. fig. 1 (A) and table 3 depicts the average size distribution of the synthesized nanoparticles using Dynamic Light Scattering zeta sizer analysis. The average particle size of ceftriaxone sodium loaded chitosan nanoparticle is $249 \mathrm{~nm}$. As shown in fig. 1 (B), the zeta potential value of ceftriaxone sodium loaded chitosan nanoparticle was $38.5 \mathrm{mV}$ which indicates the nanoparticles to be stable. To allow efficient cell interaction and promote intracellular delivery of the chitosan nanoparticulate system, a high positive zeta potential is needed [28] Zeta potential measurement is an important index in determining the stability of the nanoparticle suspension.

High absolute zeta potential value signifies high electric surface charge on the drug loaded nanoparticles, which can cause strong repellent forces among particles to prevent it from aggregation [29].

Table 3: Particle size poly dispersibility index and zeta potential of ceftriaxone sodium loaded CS-NP

\begin{tabular}{llll}
\hline Formulation code & Particle size (nm) (mean) & Poly dispersibility index (mean) & Zeta potential (mV) (mean) \\
\hline F1 & 271 & 0.021 & 42.6 \\
F2 & 249 & 0.012 & 38.5 \\
F3 & 309 & 0.213 & 46.2 \\
F4 & 311.6 & 0.417 & 47.5 \\
F5 & 345.3 & 0.434 & 44.7 \\
\hline
\end{tabular}

\section{Size Distribution by Number}

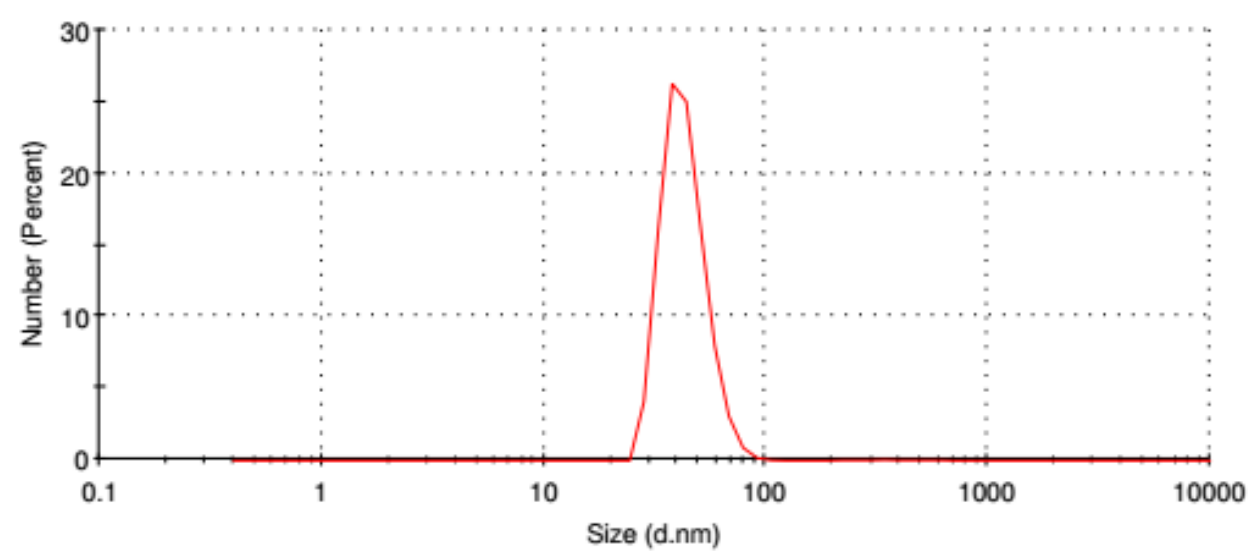

Fig. 1: (A) Particle size distribution of ceftriaxone sodium CS-NP

Zeta Potential Distribution

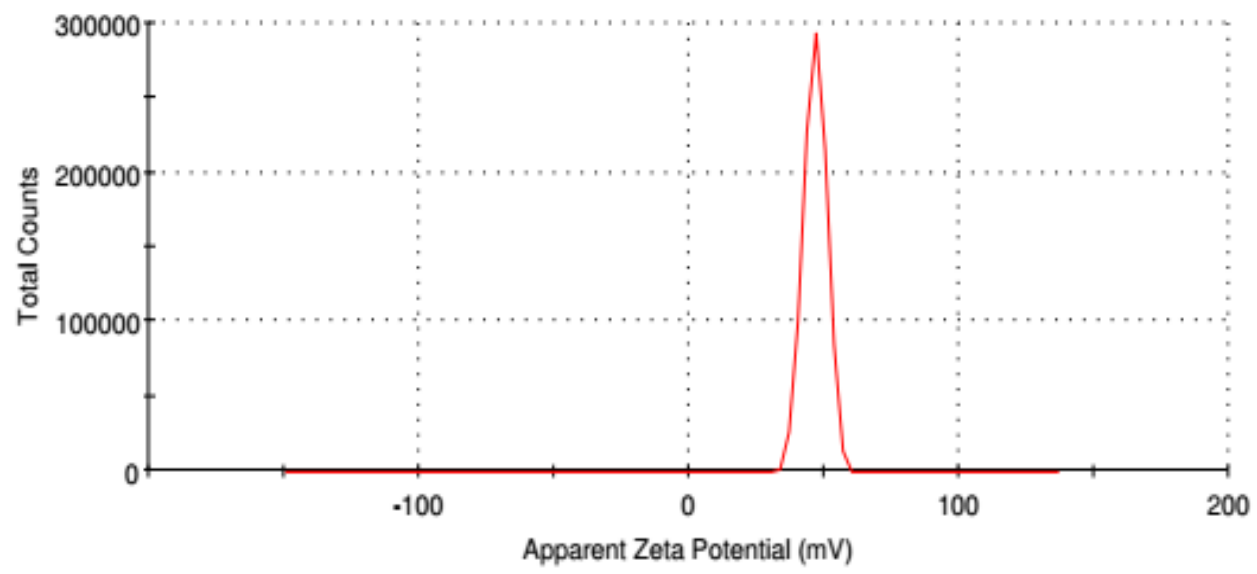

Fig. 1: (B) zeta potential of ceftriaxone sodium CS-NP

\section{In vitro drug release study}

The release profile of ceftraxone sodium loaded nanoparticles into PBS $\left(\mathrm{pH} 7.4 ; 37^{\circ} \mathrm{C}\right.$ ) are shown in table 4 , fig. 2(B). The in-vitro drug release study was performed Drug release from nanoparticles and micro particles occur by several mechanisms including surface erosion, disintegration, diffusion and desorption [21]. Ceftriaxone sodium loaded chitosan nanoparticles showed an initial release of $15.14 \%$ which may be accounted for the ceftriaxone adsorbed to the surface. Over $2 \mathrm{~h}, 83.03 \%$ of ceftriaxone sodium was released followed by slow release up to $24 \mathrm{~h}$. The increased release in aqueous media from $15.14 \%$ to $83.03 \%$ due to the swelling property of the polymer. 
Manimekalai et al.

Table 4: In vitro release profiles of ceftriaxone sodium loaded CS-NP

\begin{tabular}{|c|c|c|c|c|c|}
\hline \multirow[t]{2}{*}{ Time (min) } & \multicolumn{5}{|c|}{ Percentage of drug release for different batches of nano formulation } \\
\hline & F1 & F2 & F3 & F4 & F5 \\
\hline 0 & 0 & 0 & 0 & 0 & 0 \\
\hline 30 & $13.47 \pm 65$ & $15.14 \pm 32$ & $12.15 \pm 98$ & $14.10 \pm 32$ & $15.67 \pm 03$ \\
\hline 45 & $25.61 \pm 89$ & $27.83 \pm 25$ & $22.40 \pm 43$ & $26.40 \pm 25$ & $28.19 \pm 68$ \\
\hline 60 & $32.29 \pm 54$ & $36.92 \pm 52$ & $26.80 \pm 62$ & $31.90 \pm 21$ & $33.26 \pm 14$ \\
\hline 120 & $40.45 \pm 74$ & $47.09 \pm 45$ & $32.00 \pm 51$ & $39.00 \pm 24$ & $40.21 \pm 19$ \\
\hline 240 & $47.08 \pm 25$ & $55.64 \pm 75$ & $36.20 \pm 75$ & $46.30 \pm 36$ & $49.91 \pm 07$ \\
\hline 360 & $52.93 \pm 21$ & $62.75 \pm 95$ & $40.50 \pm 64$ & $53.20 \pm 37$ & $57.24 \pm 15$ \\
\hline 480 & $58.68 \pm 65$ & $68.65 \pm 12$ & $46.10 \pm 85$ & $57.40 \pm 96$ & $64.11 \pm 62$ \\
\hline 600 & $65.18 \pm 78$ & $72.88 \pm 23$ & $49.20 \pm 53$ & $60.20 \pm 85$ & $69.64 \pm 35$ \\
\hline 720 & $71.38 \pm 85$ & $81.10 \pm 42$ & $54.50 \pm 35$ & $63.80 \pm 67$ & $73.93 \pm 75$ \\
\hline 1440 & $72.58 \pm 25$ & $83.08 \pm 63$ & $57.00 \pm 43$ & $66.80 \pm 61$ & $77.34 \pm 42$ \\
\hline
\end{tabular}

Data's are expressed as mean $\pm \operatorname{SEM}(n=3)$

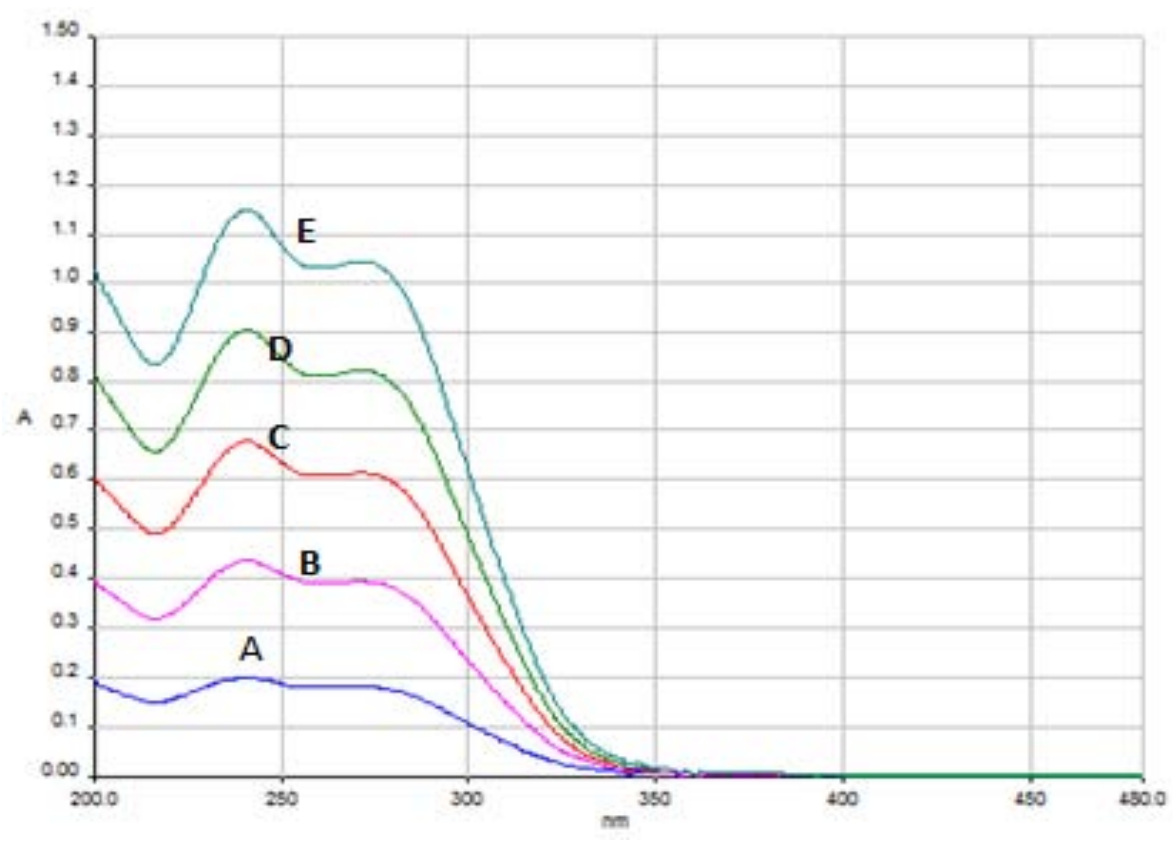

Fig. 2: (A) UV-visible spectrum of A-F1, B-F2, C-F3, D-F4, E-F5

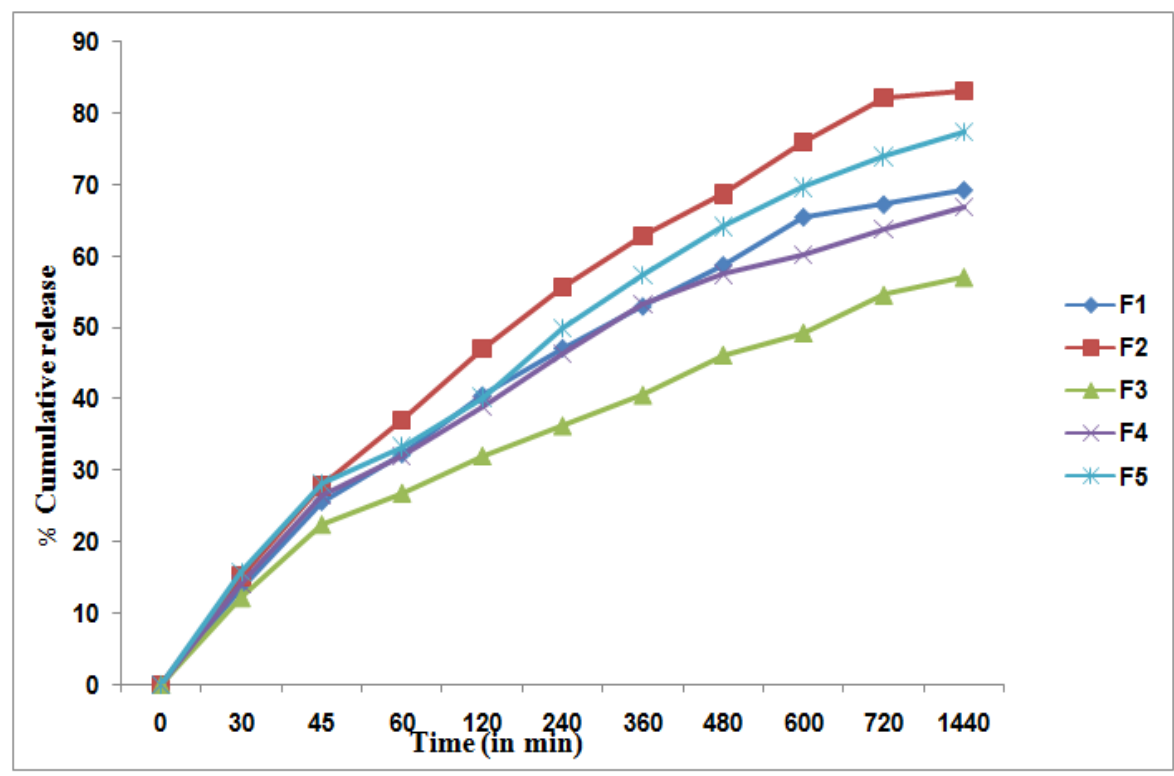

Fig. 2: (B) In vitro drug release profile for the formulation of ceftriaxone loaded CS-NP 


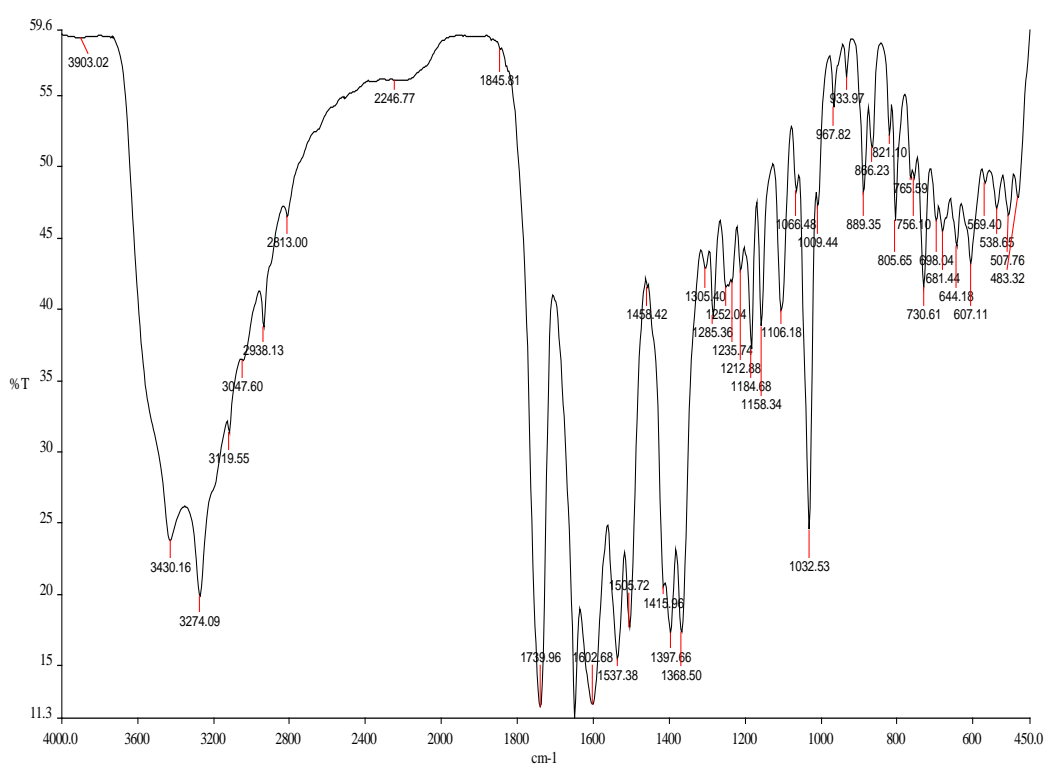

Fig. 3: FT-IR spectrum of ceftriaxone sodium loaded-CS-NP

FTIR studies for Ceftriaxone showed (fig. 3) characteristic peaks at $3432.7 \mathrm{~cm}^{-1}(\mathrm{~N}-\mathrm{H}$ stretching mode of $\mathrm{H}$-bonded amide group), $1741 \mathrm{~cm}^{-1}$ ( $\beta$-lactam $\mathrm{C}=0$ stretching vibrations) and $1592 \mathrm{~cm}^{-1}$ oxime $\mathrm{C}=\mathrm{N}$ stretching vibrations). The spectrum of pure chitosan exhibited an amine deformation peak at $1600 \mathrm{~cm}^{-1}$ and amide I carbonyl stretch at $1643 \mathrm{~cm}^{-1}$ (Samuels, 1981). The IR spectral interpretation shows that the spectra obtained from the formulation matches with original spectra of drug. Similarly characteristic peaks, for the polymers were also noticed in the formulation spectrum. There was no change of any characteristic peaks which confirms that the absence of chemical interaction between the drug and polymers.

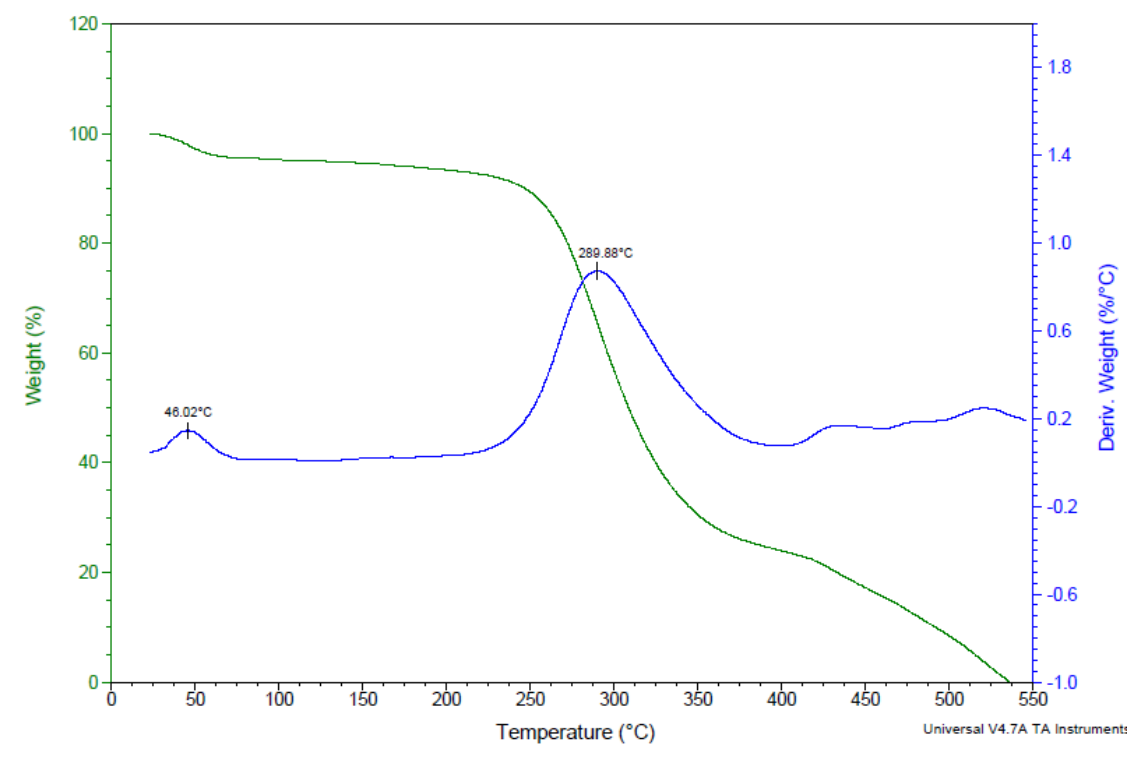

Fig. 4: Thermo gravimetric analysis of ceftriaxone loaded CS-NP

The thermogravimetric analyzer (TGA) is an essential laboratory tool used for material characterization. TGA is a technique in which, upon heating a material, its weight increases or decreases. Thermogravimetric analysis (TGA) is a simple analytical technique which is used to study the thermal stability of the sample and their weight loss at different temperatures. It also confirms the successful loading of ceftriaxone sodium within CS-NP. We observed a four step decomposition patterns on ceftriaxone sodium loaded CS-NP (fig. 4). Initially, $39 \%$ weight loss was observed at $40.02^{\circ} \mathrm{C}$, due to the release of water molecules. The second step in the curve is about $75 \%$ weight loss at $289.88^{\circ} \mathrm{C}$ indicating the decomposition of CS and the third step was observed at $450{ }^{\circ} \mathrm{C}$ with the weight loss of $19 \%$ could be due to the decomposition of both CS and ceftriaxone sodium. Finally the fourth step of decomposition was examined at 5
$35{ }^{\circ} \mathrm{C}$ of about $09.79 \%$ which might be due to the decomposition of pure ceftriaxone sodium. Similar decomposition pattern of ceftriaxone was also observed in solid lipid nano particle and our result correlates well with this report.

The morphology of the ceftriaxone sodium loaded chitosan nanoparticle was spherical in shape with their diameter roughly around $28 \mathrm{~nm}$ and was found to be aggregated. This is likely due to the fact that, as the particle size decreases they are liable to aggregate due to their high specific surface energy [24]. However, ceftriaxone sodium loaded chitosan fig. 5 shows distinct, spherical shaped particles with their average diameter around $30-250 \mathrm{~nm}$ This increased particle size could be due to the loading of ceftriaxone sodium chitosan nanoparticles similar results were observed in previous reports $[25,26]$. 


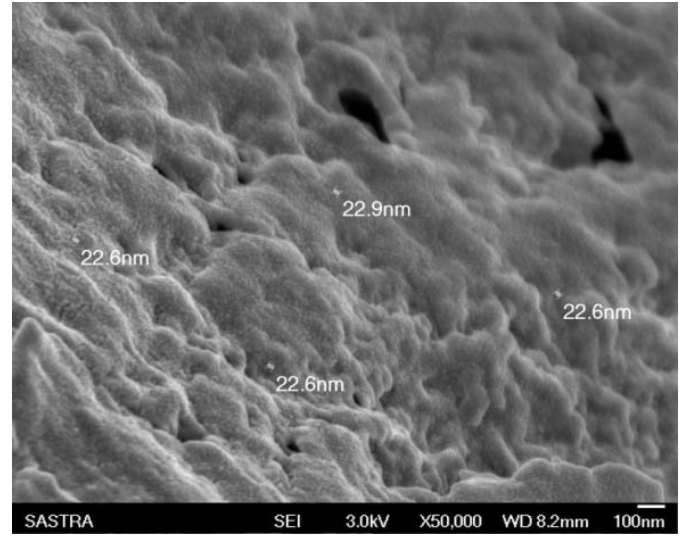

Fig. 5: SEM image of ceftriaxone sodium loaded CS-NP

\section{CONCLUSION}

We have developed a method for synthesis of ceftriaxone loaded chitosan nanoparticle using ionic cross linking method. TPP used as a cross linking agent. The final formulation ratio of drug polimer is $1 ; 2$ which act as an effective carrier with high loading efficiency. The bonding interactions between chitosan and ceftriaxone sodium were studied using FT-IR. The drug is released completely in phosphate buffer medium by $48 \mathrm{~h}$ at room temperature indicating sustained drug release pattern. SEM analysis revealed the spherical shaped morphology and the average particle size was 249 . Finally we concluded that this method is reliable and reproducible less time consuming.

\section{ACKNOWLEDGMENT}

Authors are thankful to the Anna University, Government of tamil nadu, Trichy for their technical support.

Financial support and sponsorship: Nil.

\section{CONFLICT OF INTERESTS}

\section{Declared none}

\section{REFERENCES}

1. Lipinski CA, Lombardo F, Dominy BW, Feeney PJ. Experimental and computational approaches to estimate solubility and permeability in drug discovery and development settings. Adv Drug Delivery Rev 2001;46:3-26.

2. Lee S, Kim SK, Lee DY, Park K, Kumar TS, Chae SY, et al. Cationic analog of deoxycholate as an oral delivery carrier for ceftriaxone. J Pharm Sci 2005;94:8-18.

3. Cho SW, Lee JS, Choi SH. Enhanced oral bioavailability of poorly absorbed drugs. I. Screening of absorption carrier for the ceftriaxone complex. J Pharm Sci 2004;93:612-20.

4. Shanmugarathinam A. Puratchikody, formulation and characterisation of ritonavir loaded ethylcellulose buoyant microspheres. J Pharm Sci Res 2014;8:274-7.

5. Gan Q, Wang T, Cochrane C, McCarron P. Modulation of surface charge, particle size and morphological properties of chitosan TPP nanoparticles intended for gene delivery. Colloids Surf B 2005; 44:65-73.

6. Ishak RAH, Awad GAS, Mortada ND, Nour SA. Preparation, in vitro and in vivo evaluation of stomach specific metronidazole loaded alginate beads as local anti-helicobacter pylori therapy. J Controlled Release 2007;119:207-14.

7. Rao JP, Kurt E, Meckler KE. Polymer nanoparticles: preparation techniques and size-control parameters. Prog Polym Sci 2011;36:887-913.

8. S Sakhare, AV Yadav, PD Jadhav. Design, development and characterization of mucoadhesive gastro spheres of carvedilol. Int J Appl Pharm 2016;3:37-42.
9. Hu FQ, Meng P, Dai YQ Du YZ, You J, Wei XH, et al. PEGylated chitosan-based polymer micelle as an intracellular delivery carrier for anti-tumor targeting therapy. Eur J Pharm Biopharm 2008;70:749-57.

10. Chen $\mathrm{X}, \mathrm{WJ} \mathrm{Li}$, TY Yu. Conformation transition of silk fibroin induced by blending chitosan. J Polymer Sci B 1997;35:2293-6.

11. Chiou WL, Riegelman S. Pharmaceutical applications of solid dispersion systems. J Pharm Sci 1971;60:1281-302.

12. El-Shabouri MH. Positively charged nanoparticles for improving the oral bioavailability of cyclosporine. Int J Pharm 2002;249:101.

13. Venkateswarlu V, Manjunath K. Preparation, characterization and in vitro release kinetics of clozapine solid lipid nanoparticles. J Controlled Release 2004;95:627-38.

14. Moghimi SM, Hunter AC, Murray JC. Nanomedicine: current status and future prospects. FASEB J 2005;19:300-11.

15. Bretschneider B, Brandsch M, Neubert R. Intestinal transport of beta-lactam antibiotics: analysis of the affinity at the $\mathrm{H}+$ /peptide symporter (PEPT1), the uptake into Caco-2 cell monolayers andtransepithelial flux. Pharm Res 1999;16:55-61.

16. Nasti A, Zaki NM, de Leonardis P, Ungphaiboon S, Sansongsak P, Rimoli MG, et al. Chitosan/TPP and chitosan/TPP-hyaluronic acid nanoparticles: systematic optimisation of the preparative process and preliminary biological evaluation. Pharm Res 2009;26:1918-30.

17. J Joysa Ruby, VP Pandey. Chitosan nanoparticles as a nasal drug delivery for memantine hydrochloride. Int J Pharm Pharm Sci 2015;1:34-7.

18. Giron D. Application of thermal analysis in the pharmaceutical industry. J Pharm Biomed Anal 1986;4:755-70.

19. Y Kawashima, T Handa, A Kasai, H Takenaka, SY Lin. Novel method for the preparation of controlled-release theophylline granules coated with a polyelectrolyte complex of sodium polyphosphate-chitosan. Chem Pharm Bull 1985;33:2469-74.

20. P Manimekalai, R Manavalan. Selection of excipients for the formulation of ceftriaxone sodium loaded chitosan nanoparticle through drug-excipient compatibility testing. Int J PharmTech Res 2015;8:5-10.

21. P Manimekalai, R Manavalan. Moleculardocking studies of ceftriaxone sodium with apoptosis protein in colorectal cancer. Int J Res Pharm Sci 2014;5:250-5.

22. Elizabath Antony, Mythili Sathiavelu, Sathiavelu Arunachalam. Synthesis of silver nanoparticles from the medicinal plant bauhinia acuminata and biophytum sensitivum-a comparative study of its biological activities with plant extract. Int J Appl Pharm 2017;9:22-9.

23. Pannerselvam, Balashanmugam, Pudupalayam T, Kalaichelvan. Biosynthesis characterization of silver nanoparticles using cassia roxburghii DC aqueous extract and coated on cotton cloth for effective antibacterial activity. Int J Nanomed 2015;10:87-97.

24. Arulmozhi K, Pandian S, Mirunalinia ellagic acid encapsulated chitosan nanoparticles for drug delivery system in human oral cancer cell line (KB) 3. Colloids Surf B 2013;110:313-20.

25. Ohya Y, Shiratani M, Kobayashi H, Ouchi T. Release behaviour of 5-fluorouracil from chitosan-gel nanospheres immobilizing 5-fluorouracil coated with polysaccharides and their cell specific cytotoxicity. Pure Appl Chem 1994;31:629-42.

26. Lifeng Qi, Zirong Xu, Xia Jiang, Caihong Hu, Xiangfei Zou. Preparation and antibacterial activity of chitosan nanoparticles. Carbohydrate Res 2004;339:2693-700.

27. Simar Preet Kaur, Rekha Rao, Afzal Hussain, Sarita Khatkar. Preparation and characterization of rivastigmine loaded chitosan nanoparticles. J Pharm Sci Res 2011;5:1227-32.

28. Elkholi IE, Hazem NM, ElKashef WF, Sobh MA, Shaalan D. Evaluation of anti-cancer potential of capsaicin-loaded trimethyl chitosan-based nanoparticles in HepG2 hepatocarcinoma cells. J Nanomed Nanotechnol 2014;5:240-5.

29. Anbarasan B, Vennya V Menon, Niranjana VA, Ramprabhu. Optimization of the formulation and in-vitro evaluation of chloroquine loaded chitosan nanoparticles using ionic gelation method. J Chem Pharm Sci 2013;6:407-12. 